\title{
Critical Evaluation of the Cloud-Based Design and Manufacturing
}

\author{
${ }^{1}$ Abirami S K and ${ }^{2}$ Keerthika J \\ ${ }^{1}$ Department of Computer Science and Engineering, Sri Eshwar College of Engineering, Coimbatore, India. \\ 1abirami.s.k@sece.ac.in, ${ }^{2}$ keerthika.jcse@sece.ac.in
}

\begin{abstract}
ArticleInfo
Journal of Machine and Computing (http://anapub.co.ke/journals/jmc/jmc.html)

Doi : https://doi.org/10.53759/7669/jmc202101019

Received 20 March 2021; Revised form 28 June 2021; Accepted 10 August 2021; Available online 05 October 2021. (C)2021 The Authors. Published by AnaPub Publications.

This is an open access article under the CC BY-NC-ND license. (http://creativecommons.org/licenses/by-nc-nd/4.0/)

Abstract - This article examines Cloud-based Design and Manufacturing from a critical standpoint (CBDM). Cloud technology has lately found its way into the realm of computer-assisted product creation. Corporations could explore substituting existing own CAD software licences with Design software as a cloud - based service as the first part of implementation. Installing a CAD program via the cloud on a carrier's server and incurring a miniscule proportion of the initial licensing price on a pay-per-use basis is definitely tempting. Furthermore, time and money-consuming software upgrades and operational issues are no longer an issue. We provide an introduction of cloud technology and the intrinsic features that drive its usage in both the business and education domains for dispersed and interactive design and production. Cloud Technology is a hotly debated Information Technology (IT) model that is expected to have a major effect on how businesses are run in the future. While cloud technology was first proposed in the late 60s, it was only recently that it became a viable part of day-to-day IT systems, thanks to the Internet's increasing prevalence and other modern improvements in information communication technology (ICT).
\end{abstract}

Keywords - Cloud-based Design and Manufacture (CBDM), Information Technology (IT), Computer-Assisted Design (CAD)

\section{INTRODUCTION}

The New paradigms of corporate business systems and design construction procedures are being induced by the World Wide Web (WWW), Globalization and the Internet. Competitiveness, unparalleled rates of science and technology exploration and design, automatic distribution of knowledge through the use of Internet communications technologies, vast amount of data and information, and ever-increasing variability of engineering processes and their design concepts, all combined with an insatiable desire to reduce time-to-market while optimizing product quality. These are only a few of the variables influencing the worldwide procurement and supply environment, as well as the sectors and businesses that support it. Internet technology solutions and their data and communications paradigms such as social media, digital multiple access video tele-collaboration, Voice Over Internet Protocol (VoIP), chat rooms, cloud-based communities, virtual social worlds, and many others have made it easier for users from all areas of life from all over the world to work together seamlessly [1].

Globalization, mass cooperation, mass customisation, and also new technologies like cloud computing and virtualization, have all been made possible by these innovations. These technologies enable clear interaction on a variety of platforms, including cellphones, netbooks, computers, and desktops. The above-mentioned Web information and communications technology (ICT) have also offered methods for fundamentally new techniques of invention, research, design, and production that could not have been envisaged even a few years ago. Mass cooperation, distributed development, and distributed manufacturing, in particular, are now feasible methods for the next generations of products design and production, thanks to the Internet [2]. The approach, which we call Collaboration and Decentralized Development and Manufacturing (CDDM), is capable of encompassing the full product lifecycle that centers on the manufacturing process.

Product conceptualization, development, understanding, evaluation, synthesis, simulations, modeling, and dominant process are all part of the product lifecycle. This isn't to imply that the "Web" and "Internet" are capable of carrying out these tasks. Conversely, the Internet and Web offer ICT frameworks and solutions that connect the many elements of the fundamental production lifecycle infrastructure necessary for the operations. CDDM offers greater economies of scale, a faster time to market, and a variety of additional benefits to industrial companies. Many conventional businesses that concentrate in product design and production use a decentralized and centralized unit structure. As a result, this 'vertical structure' of business divisions contains homogeneous advanced engineering groups with their pillars of separated design processes. Today's goods, on the other hand, are fully interconnected complex machines made up of technology families that span many layers and domains. As a consequence, multidisciplinary engineering design is necessary and must be integrated into the product development process.

Discrete silos of advanced engineering techniques must be combined (combined) into heterogeneous multidisciplinary engineering design units to meet this need. The fact that almost all, if not all, elements of a vertical integration business 
(VIB) are 'under one roof' is one of its distinguishing characteristics [3]. The product families of the VIB are created, developed, produced, marketed, and distributed by the company's assets. In many areas, however, goods have grown more complicated and technologically advanced in both design and production. The capital expenditures required to realize diverse VIB product portfolios may become prohibitive. When product categories based on technology develop at a rapid pace, such as the semiconductors and embedded processors, this occurs. As a consequence, the VIB is increasingly becoming the exception rather than the norm.

Rather, today's common enterprise - the Internationalization 3.0 venture, or G3E - is founded on the principles such as IP centricity (intellectual property centricity), bilateral agreement, mass cooperation, lean manufacturing, fabrication-less merchandise fulfillment, decentralized configuration, decentralized production, decentralized manufacturing, dispersed distribution channels, and so on. G3 has made it possible for businesses to have dispersed 'assets,' such as human, structural, and digital assets. Design-by-collective-intelligence may become a widespread design approach in the near future, fueled by crowd-sourced development, mass cooperation, and mass customisation. Rather than vertical integration businesses, fuzzier supply-demand networks are anticipated to develop, with the concept of VIP families gaining traction.

The physical distribution of engineering skills matter specialists within a group of separate businesses establishing strategic alliances and pooling resources to accomplish a shared goal, including the realization of products-is used to create a VIP family. The implementation of G3E products will need a change in current cross-disciplinary architectural layout and layout integration technology [4]. The initial cross-disciplinary designs integrated study questions, aims, goals, and assumptions will generate more sophisticated versions when cross-disciplinary design integration is required across organizational boundaries. Not only will cross-disciplinary continuous design inter organizational borders, but it will also transcend barriers in the so-called "Clouds." Future industrial engineering and fabrication procedures would be crossenterprise and cross-disciplinary in design, and the Cloud will allow, or at the very least substantially promote these activities. Resultantly, industry practitioners and researchers are constantly looking for more efficient and appropriate manufacturing and designing methods to help engineers capture and reuse data and insights, as well as management must ensure that diverse business and engineering processes are coordinated across individual and organisational cooperation and collaboration [5].

Resultantly, we believe that for future research and development and implementation procedures, a cloud-based engineering and manufacturing methodology is required. While the previous has primarily been presented from the standpoint of cutting-edge investigation underneath the framework of sophisticated design and engineering, it is also critical to incorporate an appropriate dose of our investigation into our learning programmes and expose our trainees to the most recent advancements. As a result, we've integrated our cloud-based development and manufacturing infrastructure, as well as associated technologies, into one of our postgraduate advanced engineering courses. An engineering and industrial production open-ended issue is a major component of this course. This issue will be addressed by a group of around 30 students from throughout the nation, who will use ideas like crowd sourcing, mass collaborations, and cloud-based development and construction, among other things. The background assessment of the cloud computing technology is provided in Section II below. Section III provides a critical analysis of the paper with major focus on Cloud-Based Design and Manufacture (CBDM). Lastly, Section IV concludes the paper.

\section{BACKGROUND ANALYSIS}

Paradigm of Cloud Computing

This section provides an overview of cloud technology and the underlying qualities that encourage its usage in both the industrial and educational domains for remote and cooperative design and manufacturing. Cloud Technology is a hotly debated Information Technology (IT) concept that is expected to have a big influence on the future of enterprise. Whereas cloud technology was first proposed in the 1960s, it was only recently that it became a viable part of day-to-day IT systems, thanks to the Web's widespread availability and other recent advances in information and computer technology. However, no one description for cloud technology exists at this time, since scholars, business leaders, and government organizations have all attempted to define it based on their own objectives, responsibilities, and objectives. In general, cloud technology is dedicated to providing technology as a fully managed service, in which shared facilities, applications, and data are delivered as a commodity across the internet to computer devices. In other terms, it's a sophisticated advertising phrase for a group of computer networks that deliver services (or assets) to a group of customers through the WWW.

Software as a service (SaaS), platform as a service (PaaS), and infrastructure as a service (IaaS) are the three most important layers presently defining cloud technology [6], as seen in Fig 1. Because of ubiquitous and dependability of Internet connections, sophisticated Web 2.0 capabilities, the lower cost of entrepreneurship virtual machines, and other auxiliary innovations like application devices and internet emulation, these three levels of service are theoretically feasible.

Customers can access software programs over the website, removing the requirement to download and operate programs on their own pcs. The two major benefits of this strategy are cost savings from a pay-as-you-go strategy vs. complete software license, and no delay for system support and maintenance. Clients can get a complete desktop workstation, including network infrastructure and cloud - based services, as a platform as a service. As a result, it simplifies program installation by removing the expense and burden of purchasing and maintaining the software and hardware levels.

Clients generally receive a virtual computing ecosystem, as well as networking and storage abilities, as part of Cloud infrastructure [7]. Users lease computers, applications, and networking devices as a completely external provider on a payas-you-go model rather than acquiring them. The fog, which has been de-mystified, can be governmental, personal, or mixed in form. In other instances, businesses (or clients) may opt to develop their cloud systems as LAN (Local Area 
Network), utilize the $3^{\text {rd }}$ cloud framework (cloud computing), or use a mix - for example, renting and running Software as a Service within the local network system while storing implementation data in a single, cloud system (see Fig. 2).

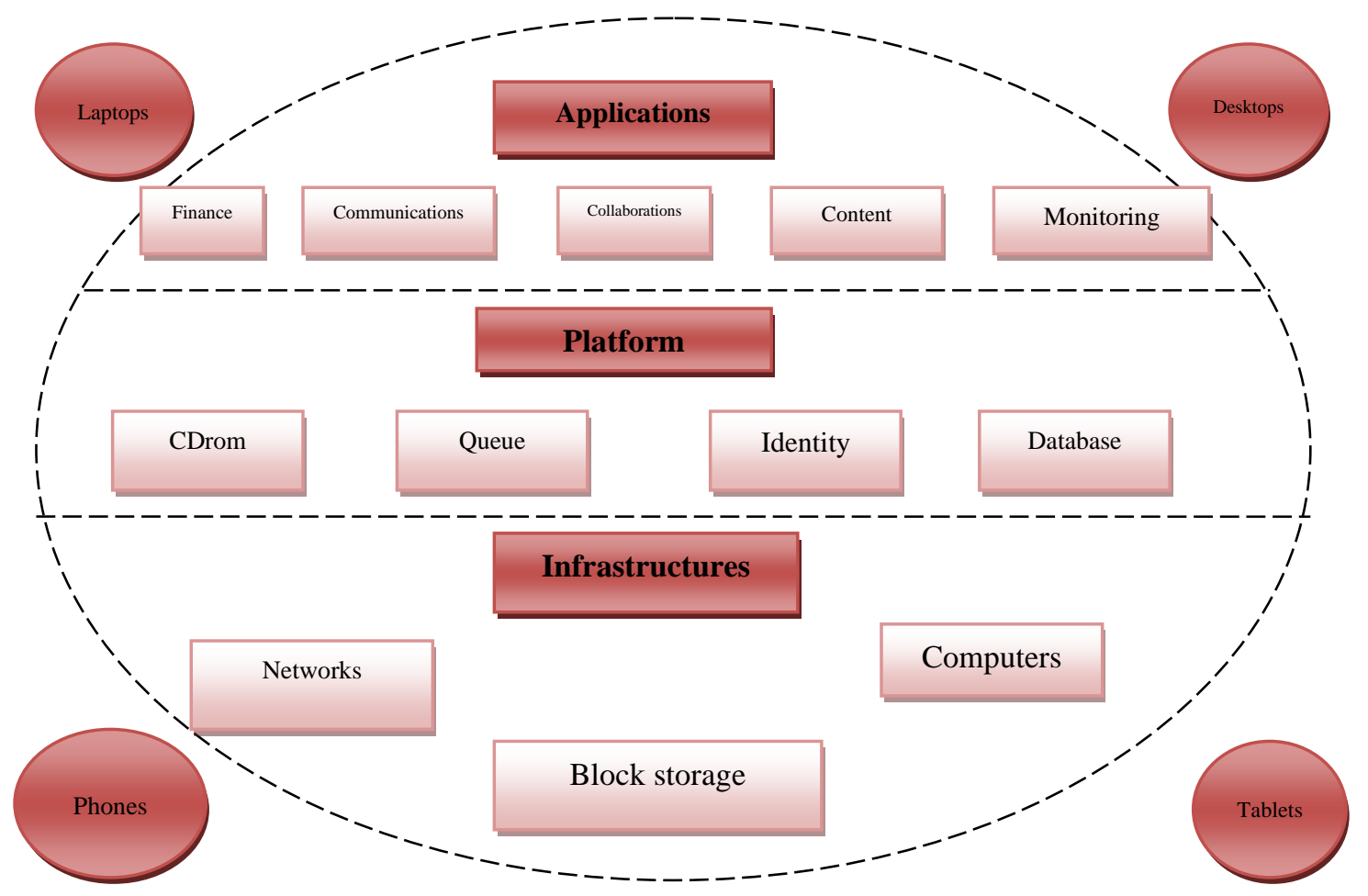

Fig 1. Cloud computing service tiers

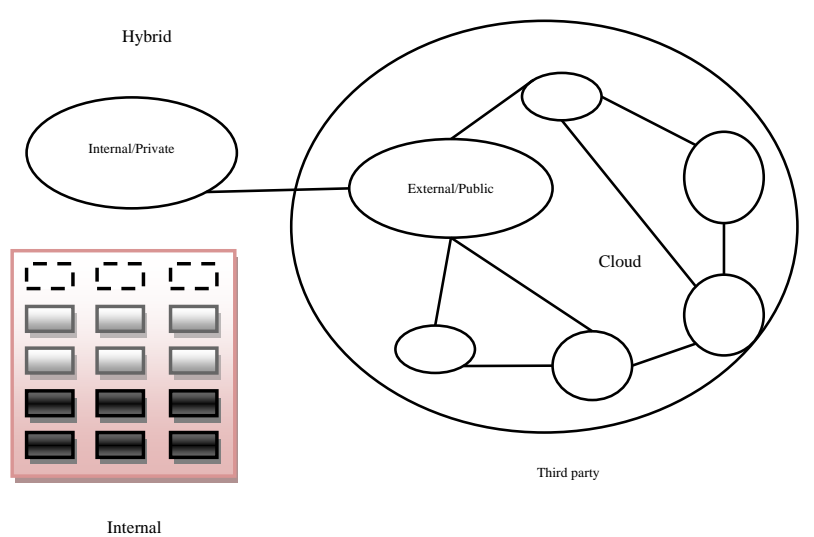

Fig 2. Types of cloud computing

SaaS is the most popular cloud computing service right now, followed by PaaS and IaaS. Because public cloud is still in its development, it's no surprise that it's most commonly used in the IT industry right now. Other industries, on the other hand, have begun to see its usefulness on a wider scale, and substantial consequences are expected [8]. For instance, Narasimhan and Nichols, in collaboration with Itracks, an internet market research organization, conducted a study of 155 firms that have used cloud computing services and discovered the following findings: (1) 60 percent of study respondents favor cloud computing over on-premise deployments, and (2) 68 percent expect to put a substantial percentage of their programs in cloud computing within three years after the poll. In their article, they provide additional statistical information. The framework of virtualized design and production, which is discussed in the next section, is one example of a fog processing appliance that has started to emerge. 
Cloud-Based Design and Manufacture (CBDM)

Public cloud has lately found its way into the pc product creation field. Organizations could explore substituting their own CAD software licences with CAD software as a cloud - based service as a first move in this approach. Operating CAD software over the fog on a supplier's database(s) and charging a decent percentage of the initial licensing cost on a pay-peruse system is very attractive. Furthermore, time-consuming and costly software upgrades and service concerns are no longer an issue. On the negative, an individual local network device enables far quicker data transmission speed than an Internet access. Furthermore, displaying CAD information can be highly important in terms of computer power, and there may be a little latency in reaction time when using the Web [9]. The numerous use situations will determine whether or not latency is reasonable. While this may be tolerable in a CAD learning setting, displaying delays due to latency can be inconvenient (and expensive) in a complete design process. In practise, most businesses choose to run their program remotely since it is faster.

The storage of CAD software applications on the same website is one method to reduce response time. The less information that must be sent over the fog, the faster and faster/less expensive it might be. As a result of this, and as mentioned in Section I, supplying data storage as a system via the fog is a viable business model to take into account. Storage of data on a pay-as-you-go basis may be significantly less expensive than acquiring your own hard disk drives, and, as with SaaS, device preservation and substitution are no longer a problem. The safety of data is a major concern when using cloud technology. Identifying where valuable data is kept in the fog and what will occur to it in the event of a power outage or system disaster is a big worry for any organization. Nevertheless, the total chance of data loss is negligible. Substantial, well-known virtualized providers generally spell out all information security and IP-related clauses in their contracts, as well as give testimonials from high-profile customers with extremely private data. Other marketing 'everything-as-a-service' designs have begun to develop in relation to CAD cloud infrastructure.

The 'mobile Network,' which will be one specific communication technology that supports ubiquity cloud technology for portable 'cell devices' apps, as well as smart phones actually delivering fog functions such as data gathering, i.e. mobile phone detectors, will follow the same path. In [11], for instance, estimated that the number of mobile Phone users may reach ten times that of pcs Online consumers. While computer and information systems undergo a tremendous paradigm change from the old customer prototype to the improved cloud computing platform, the design and production community are beginning to incorporate public cloud elements. This concept is being tried by a number of firms, notably Autocad and Fujitsu. Autocad, for instance, promises to be able to give consumers with improved access to construction and technical documentation at any time and from any location [10]. The following are some of the highlighted facilities:

- Cloud modeling, which provides clients with strong processing abilities to improve 3D model representation; and

- Software-as-a-Service, which enables designers to securely communicate information to improve teamwork efficiency and effectiveness.

Fujitsu's design fog, for instance, allows for the effective consolidation of applications such as high data types. Their research fog offers a high-performance 'thin clients' atmosphere, as well as system and licensing aggregation. The prospect of expanding the cloud computing technology to material assets such as 3D scanners for decentralized and Network advanced manufacturing equipment such as milling, saws, and other industrial production facilities is one emerging paradigm now under investigation. In the long run, virtualized digital project management (which ambition, assessment, and modeling, as well as production) is projected to be the norm.

It's being hailed as a new paradigm for assisting upcoming internationally dispersed design and production operations that effortlessly combine both physical and virtual assets, such as CAD programs and aggregate manufacturing equipment. Fog Design and Production is the name given to this new approach and emerging concept (CBDM). CBDM is a product innovation paradigm that aims to provide on-demand sharing of resources and flexibility by utilizing product design and production major mechanism through cloud service software and hardware [12]. As a result, for internet design and production, we suggest the following concept: Internet Design and Production is a production process approach that uses networking sites and community channels, as well as managed service groups of architecture, production, and constituent capabilities, to allow collaborative innovation process and quick product creation with minimal expenses. Fig 3 depicts the ideas that underpin the fundamentals and ideas of CBDM platforms as defined by our proposal.

It's important noting at this point how the word "fog" is used. Fog images have long been used by telecommunication and network operators to represent the underlying interconnectivity complex of systems. A system of any acceptable scale is, in general, too complicated to depict on a map. As a result, fog maps are utilized to disguise the interconnection intricacy while showing the most important aspects of a network design.

As shown in Fig 3, the Online communication 'fog' serves as the fundamental and necessary 'underlay' connection for every CBDM network. CBDM technologies, as earlier said, are supported by digital communication and information technologies. Fig 3 depicts this reliance by depicting CBDM as a 'overlay.'

Fig 3 also aims to depict the general and fundamental interconnectedness of the key parts of a CBDM system. For instance, a CBDM program's for peoples resources so as to create their own "human-centric networking," which includes design engineers, social networking sites, and learners, to mention a few. Similarly, the fog nodes, which comprise people, digital, and material resources, are depicted with their corresponding 'dividers.' One of CBDM's main aims is to make product innovation and implementation more convenient. As a result, suitable linkages are formed between this aim and 
the diagram's fundamental divisions. Furthermore, the 'needs' of the product's creation and manifestation process, specifically business and education demands, should be observed.

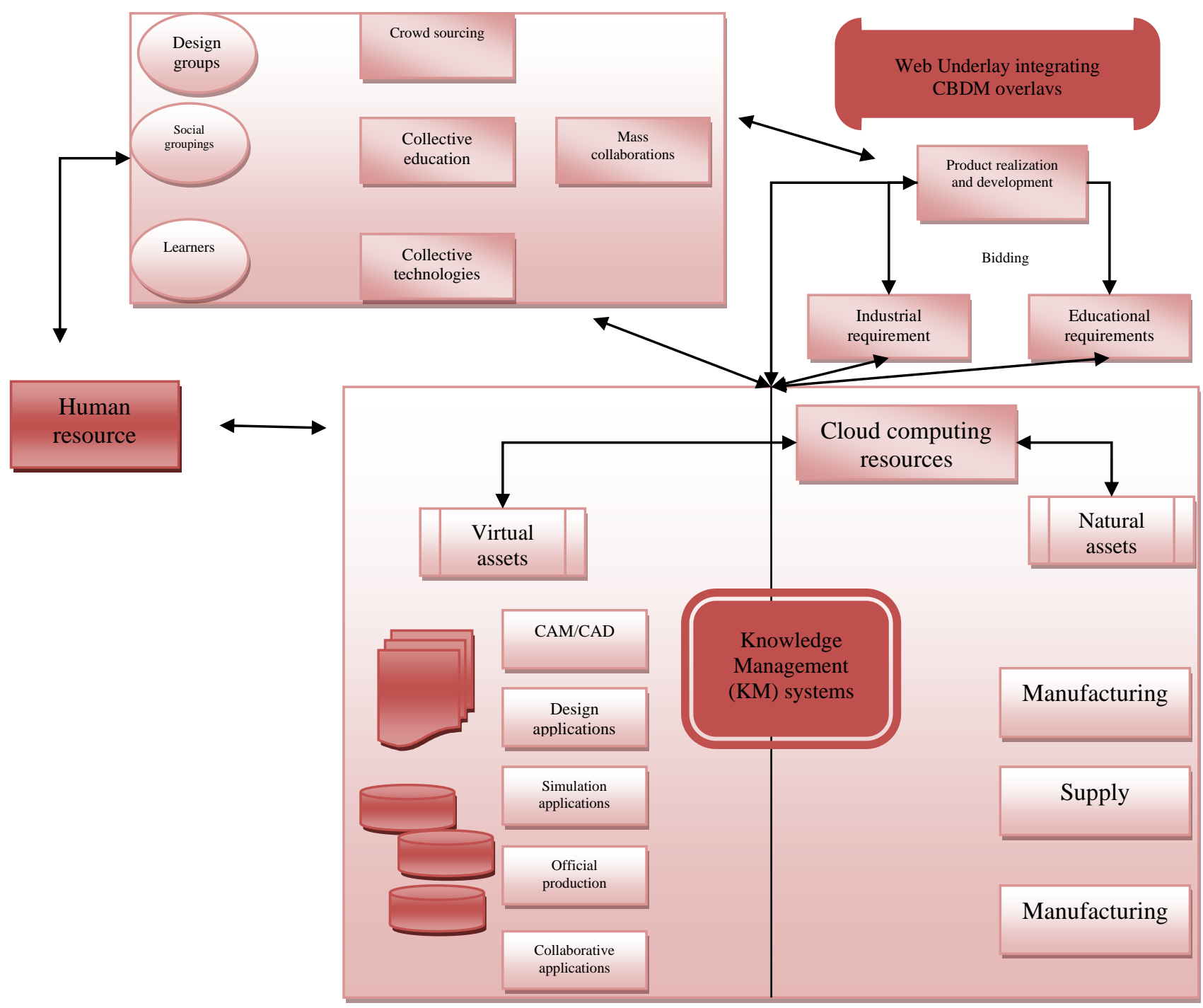

Fig 3. The concept of CBDM network

These two industries represent the most basic types of entities that require CBDM capabilities. Furthermore, the demands of industry and education are inextricably linked. CBDM technologies will be used by industries to generate raw materials and services. Clearly, industry relies on educational institutions to (1) train learners on the fundamental concepts and foundation of the CBDM networking system to accomplish the stipulated aims and objectives, and (2) consider undertaking sophisticated researchers and innovation regarding the critical CBDM networking aspects. As a result, the education and business sectors are inextricably linked.

\section{CBDM Infrastructure}

A matching architect must be created as a first stage towards fulfilling our aims and ambitions for CBDM. In order to establish an adequate basis that reflects our concept of CBDM structures, Scholars in [13] have extended their expertise on distant labs to the fields of distant hardware and software components during the past two decades. The subtopics that followed provide a description of the CBDM architecture as well as a model application.

A Cloud Computing-based Architecture for Remote CBDM

Architecture, generally, represents the gathering of resources e.g. the human capital, natural resources, operational procedures, and organization structure that are necessary to achieve a certain set of goals. For instance, a nation's transportation system makes it easier to supply raw materials, which are subsequently utilized to make commodities, which are then supplied to customers. One may naively believe that the road system is merely a government's network of roads. The transportation system, on the other hand, is more complicated than merely the road system. Rather, it is made up of the highway communication network, the systems of organisations that create raw resources, the mechanism of companies that 
manufacture products from the industrial materials, the organization that distribute the goods and basic products, and the end product users. It is simple to claim that architecture is a complicated "Network of Networks."

One notion inherent to all architecture is that its network of properties is used to bring together issue owners and hard workers in order to generate an outcome that aids the remedy to the basic requirement suggested by the infrastructure's need. Architecture is a group (system) of commodities that work together to achieve a set of goals that no one asset could achieve on its own. The connectivity of its resources, which is the link amongst issue holders and critical thinkers, determines the value contributed by the architecture.

All user interface elements (such as Internet browsers), communication and safety aspects (such as the Web and business gateway networks), personal resources (such as customers, workers, buyers, or supervisors), and real production process of assets are all included in DICIS. Manufacturing process assets (MPAs) comprise both computer and physical aspects including 3D printers, steel mills, electronic prototype devices, and automated machinery. Despite the fact that a "natural" cloud services architecture often only depicts software packages, the DICIS concept for CBDM integrates digital resources such as computer elements and programs; and physical and human resources (i.e. the apparatus described previously). The DICIS concept and its deployment as a CBDM platform may be thought of as an interconnected design and production architecture that can serve both business and education purposes, such as digital science lab courses and investigation.

CBDM resources are classified into three categories per the DICIS prototype: Human Resources, Communication Resources, and Production Process. Resources are the three types of commodities, additionally, both the Central Interface (CI) and the disseminated infrastructures are connected to personal, information, and industrial reference tools (DI). The CBDM's main physical, digital, and human capitals are all housed in the decentralised network. The centrally controlled device, on the other hand, provides the support that holds the framework together. It consists of two primary organizations of elements: User's Interface Component (UICs) and the Management's Interface Components (MICs). The DICIS model takes into account three types of human assets: service customers, service providers, and care managers. CBDM service users make use of the CBDM's resources. Students involved in decentralized design and production initiatives, scientists researching latest design concepts, or firms with globally scattered production facilities that need to produce the materials of a new device are all samples of service users [14]. Human assets in the form of intellectual labour and capital are rendered by the providers of services, hence amounting to a supply of beneficial services.

A resource provider, for instance, might be a scientific technician or manufacturing supervisor who sets a fresh range of equipment and devices within CBDM and integrates these components to formulate a novel service for customers. A distant production facility, for instance, may be building a novel three-dimension grinding and printing system within CBDM that has to be utilized by the CBDM's intellectual capital (customers). Dependent on the degree of management roles, the managers of agencies are in charge of administering the CBDM's different resources.

Create password policies, allocate role - based, schedule initiatives, install the new CBDM assets, and schedule network management, to mention some few tasks performed by management teams. Resource providers and executives, in the broadest sense, are issue responders, while service customers are issue collectors. Service providers and supervisors, on the other hand, can be problem solvers who seek the help of other service providers and supervisors. Furthermore, based on the customer's function within the network as a whole, a customer can be a resource user, provider, and/or supervisor at the same time. Take, for instance, the customer Alice. Alice may be a learner in program A, a manufacturer in program B, or a program manager in program C. DICIS' interaction resources are made up of four main parts: a communication system, an internet security device, a quality administration service communication interface (SCI), and a production process resource [15]. We postulate that the data transmission is built on the Internet Protocol (IP) and that digital interactions are normalized and widespread [16-21].

The network monitoring component encompasses the communications system aspect, reflecting both the requirement for safety and the fact that it already exists in current corporate network platforms in a variety of forms, most prominently in the case of gateway mechanisms. The quality administration SCI employs Internet techniques in order to take use of the omnipresent Web. The use of Internet mechanisms like the Hyper-Text Transport Protocol (HTTP) amongst intellectual capital and the central gateway decreases CBDM implementation costs by eliminating the need to build specific interaction programs for system use. The production process inventory SCI, on the other hand, may be more varied, and multiple methods like customer, coordination and control, and interpersonal can be utilized, based on the specific needs of a component of the CBDM. The DICIS model's production process components include equipment (tangible) and computer (digital) design and production assets. Our present CBDM, which is based on the DICIS paradigm, comprises of a hybrid software and hardware environment that supports industrial and scientific hardware components such as machining processes, saws, laser printers, 3D scanners, and do-it-yourself using 3D printing technology.

\section{CONCLUSION}

We have provided an analysis of our efforts to extend the cloud computing paradigm to the area of industrial applications in this article. A workable concept and first concept for Cloud Based Design and Manufacture (CBDM) have been proposed, as well as an IT infrastructure to enable our CBDM research efforts. Furthermore, we have described how we are using CBDM study in the area of a remote education advanced engineering program. In terms of future work, a framework detailing important research problems related to CBDM implementation and its effect on the computer-assisted product realization cycle is being developed. We want to study how students first react to the challenge of developing a product and producing prototypes in a distributed environment that combines virtual and physical resources, as well as how they adjust 
to it as the work plan continues. Furthermore, we want to experiment with different formats for acquainting learners with our CBDM setting, such as films, online tutorials, and related evaluation tools. At this early stage of development, it is critical to explore the instructional methods needed to expose students to the extremely rich IT ecosystem without compromising emphasis on the program's development and manufacturing particular technical aspects.

\section{References}

[1]. Y. Wu, F. He and Y. Chen, "A Service-oriented Secure Infrastructure for Feature-based Data Exchange in Cloud-based Design and Manufacture", Procedia CIRP, vol. 56, pp. 55-60, 2016. Doi: 10.1016/j.procir.2016.10.016.

[2]. Y. Wu, F. He, D. Zhang and X. Li, "Service-Oriented Feature-Based Data Exchange for Cloud-Based Design and Manufacturing", IEEE Transactions on Services Computing, vol. 11, no. 2, pp. 341-353, 2018. Doi: 10.1109/tsc.2015.2501981.

[3]. K. MacCallum, "Knowledge-based design for manufacture", Computer-Aided Design, vol. 20, no. 4, p. 224, 1988. Doi: 10.1016/00104485(88)90285-0.

[4]. E. Hui, B. Feng, C. Lee, J. Yang and J. Chen, "A design of CNC architecture based on cloud computing", Proceedings of the Institution of Mechanical Engineers, Part B: Journal of Engineering Manufacture, vol. 233, no. 4, pp. 1260-1268, 2018. Doi: 10.1177/0954405418774601.

[5]. M. An and H. Lee, "Design of the Integrated Logistics Information System Based on Cloud Computing", Lecture Notes on Software Engineering, vol. 3, no. 1, pp. 31-34, 2015. Doi: 10.7763/lnse.2015.v3.160.

[6]. N. Liu and X. Li, "Granulation-based resource classification in Cloud Manufacturing", Proceedings of the Institution of Mechanical Engineers, Part B: Journal of Engineering Manufacture, vol. 229, no. 7, pp. 1258-1270, 2015. Doi: 10.1177/0954405415572644.

[7]. N. Liu and X. Li, "Granulation-based resource classification in Cloud Manufacturing", Proceedings of the Institution of Mechanical Engineers, Part B: Journal of Engineering Manufacture, vol. 229, no. 7, pp. 1258-1270, 2015. Doi: 10.1177/0954405415572644.

[8]. C. Ventura, A. Bebamzadeh and M. Fairhurst, "Efficient performance-based design using parallel and cloud computing", The Structural Design of Tall and Special Buildings, vol. 24, no. 17, pp. 989-1001, 2015. Doi: 10.1002/tal.1222.

[9]. W. Duan, J. Zhou and K. Lai, "FSMT: a feature solid-modelling tool for feature-based design and manufacture", Computer-Aided Design, vol. 25, no. 1, pp. 29-38, 1993. Doi: 10.1016/0010-4485(93)90063-t.

[10]. H. Ismail, S. Chen and K. Hon, "Feature-based design of progressive press tools", International Journal of Machine Tools and Manufacture, vol. 36, no. 3, pp. 367-378, 1996. Doi: 10.1016/0890-6955(95)00047-x.

[11]. X. Gao and L. Li, "Design Ethics Based on Advanced Manufacture Technology", Advanced Materials Research, vol. 529, pp. 394-397, 2012. Doi: 10.4028/www.scientific.net/amr.529.394.

[12]. P. Cheng and H. Qu, "Design and Realization Based on Cloud Stack Hybrid Cloud Computing Platform", Advanced Materials Research, vol. 989-994, pp. 2297-2300, 2014. Doi: 10.4028/www.scientific.net/amr.989-994.2297.

[13]. "Cloud Based Platform Design for Court File Sharing", Computer Engineering and Intelligent Systems, 2019. Doi: 10.7176/ceis/10-3-01.

[14]. K. Vemuri, S. Oh and T. Altan, "BID: A knowledge-based system to automate blocker design", International Journal of Machine Tools and Manufacture, vol. 29, no. 4, pp. 505-518, 1989. Doi: 10.1016/0890-6955(89)90068-0.

[15]. X. CHEN, "Risk Based Design, Manufacture and Maintenance of Extreme Pressure Equipment", Journal of Mechanical Engineering, vol. 49, no. 22, p. 66, 2013. Doi: 10.3901/jme.2013.22.066.

[16]. A. Haldorai and A. Ramu, "Security and channel noise management in cognitive radio networks," Computers \& Electrical Engineering, vol. 87, p. 106784, Oct. 2020. doi:10.1016/j.compeleceng.2020.106784

[17]. A. Haldorai and A. Ramu, "Canonical Correlation Analysis Based Hyper Basis Feedforward Neural Network Classification for Urban Sustainability," Neural Processing Letters, Aug. 2020. doi:10.1007/s11063-020-10327-3

[18]. D. Devikanniga, A. Ramu, and A. Haldorai, "Efficient Diagnosis of Liver Disease using Support Vector Machine Optimized with Crows Search Algorithm,” EAI Endorsed Transactions on Energy Web, p. 164177, Jul. 2018. doi:10.4108/eai.13-7-2018.164177

[19]. H. Anandakumar and K. Umamaheswari, "Supervised machine learning techniques in cognitive radio networks during cooperative spectrum handovers," Cluster Computing, vol. 20, no. 2, pp. 1505-1515, Mar. 2017.

[20]. H. Anandakumar and K. Umamaheswari, “A bio-inspired swarm intelligence technique for social aware cognitive radio handovers," Computers \& Electrical Engineering, vol. 71, pp. 925-937, Oct. 2018. doi:10.1016/j.compeleceng.2017.09.016

[21]. R. Arulmurugan and H. Anandakumar, "Early Detection of Lung Cancer Using Wavelet Feature Descriptor and Feed Forward Back Propagation Neural Networks Classifier,” Lecture Notes in Computational Vision and Biomechanics, pp. 103-110, 2018. doi:10.1007/978-3-319-71767-8_9 